\title{
Energy Efficient Homogeneous and Heterogeneous System for Wireless Sensor Networks
}

\author{
R.Saravanakumar \\ Professor / ECE \\ PABCET.Trichy. \\ Tamilnadu.India
}

\author{
S.G.Susila \\ Visiting Faculty / ECE \\ Anna University of \\ Tech.Trichy. Tamilnadu. India
}

\author{
J.Raja \\ Registrar / Prof \& Head /ECE \\ Anna University of \\ Tech.Trichy. Tamilnadu. India
}

\begin{abstract}
In Wireless sensor Networks (WSNs), it is an important task to periodically collect data from an area of interest for time-sensitive applications. The Wireless sensor network (WSN) is a type of the wireless ad-hoc networks. It consisting of a large number of sensors is effective for gathering data in a variety of environments. The sensed data must be gathered and transmitted to a base station for further processing to meet the end-user queries. Since the sensors operate on battery of limited power, it is a great challenging aim to design an energy efficient routing protocol, which can minimize the delay while offering highenergy efficiency and long span of network lifetime. In this paper, we first completely analyzes the basic distributed clustering routing protocol LEACH (Low Energy Adaptive Clustering Hierarchy), which is a homogeneous system then proposed a new routing protocol and data aggregation method in Leachheterogeneous system which the sensor nodes form the cluster and the cluster-head elected based on the residual energy of the individual node calculation with re-clustering scheme is adopted in each cluster of the WSNs. In the Leach-Heterogeneous system the energy efficiency is increased near to $40 \%$ than LeachHomogeneous system and lifetime of the networks also increased. Simulation results using MATLAB are shows that the proposed Leach- heterogeneous system significantly reduces energy consumption and increase the total lifetime of the wireless sensor network compared to the homogeneous LEACH protocol.
\end{abstract}

\section{General Terms}

Wireless sensor networks, energy efficiency, clustering, homogeneous and heterogeneous.

\section{Keywords}

Clustering, energy efficiency, homogeneous, heterogeneous, LEACH protocol, node scheduling, network lifetime, wireless sensor networks.

\section{INTRODUCTION}

Wireless sensor network (WSN) consists of small in size sensor nodes, which form an ad-hoc distributed sensing [1] and data propagation network to collect the context information on the physical environment. WSN is widely used to collect reliable and accurate information in the distance and hazardous environments, and can be used in National Defense, Military Affairs, Industrial Control, Environmental Monitor, Traffic Management, Medical Care, Smart Home [2]-[4] etc. The sensor whose resources are limited is cheap, and depends on battery to supply electricity, so it's important for Routing to efficiently utilize its power in both military and civilian applications such as target tracking, surveillance, and security management. The sensor node has four basic components: sensing unit, processing unit, radio unit, and power unit. With their capabilities for monitoring and control, the sensors are expected to be deployed in vast area.

The main applications of sensor network is to periodically gather data from a remote terrain where each node continually senses the environment and sends back the data to the Base Station (BS) for further analysis, which is usually located considerably far from the target area. The most restrictive factor in the lifetime of wireless sensor network is limited energy resource of the deployed sensor nodes. Because the sensor nodes carry limited and generally irreplaceable power source, the protocols designed for the wireless sensor networks must take the issue of energy efficiency into consideration. Also, the network protocol should take care of other issues [5] such as self-configuration, fault tolerance, delay, etc. Another important criterion in the design of a sensor network is data delivery time since it is critical in many applications including battlefield and medical/security monitoring system. Such applications require receiving the data from sensor nodes within some time limit. Communication protocols highly affect the performance of wireless sensor networks by an evenly distribution of energy load and decreasing their energy consumption and thereupon prolonging their lifetime. Thus, designing energy efficient protocols is crucial for prolonging the lifetime of wireless sensor networks. So, we described a novel energy efficient and lifetime increased proposed protocol as enhanced from normal LEACH protocol. The remainder of this paper we introduce a novel re-clustering based data gathering approach which is having the node scheduling of active and sleep nodes in each cluster at a period of time in the wireless sensor networks to increase the lifetime with total energy consumed. The paper is organized as follows: A brief introduction with related works of LEACH protocol in homogeneous system is presented in section II. In section III describes the design of our novel proposed LEACH protocol in heterogeneous system in detail. Simulation and results are discussed in section IV. Finally, Conclusions are made in section V. 


\section{RELATED WORKS}

\subsection{Static Clustering Protocol}

In this protocol, the sensor nodes from the entire network is shown in fig. 1, are divided into several clusters, cluster-head nodes communicate with the local base station, then the local base station feed data to the entire network of base stations, and terminal user can access useful information. The distance between the local base stations and the cluster node was very close, therefore greatly reducing the energy consumption of these nodes send their information to local base station. In view of this, static clustering protocol seems to be a more efficient communication protocol [6]-[9].

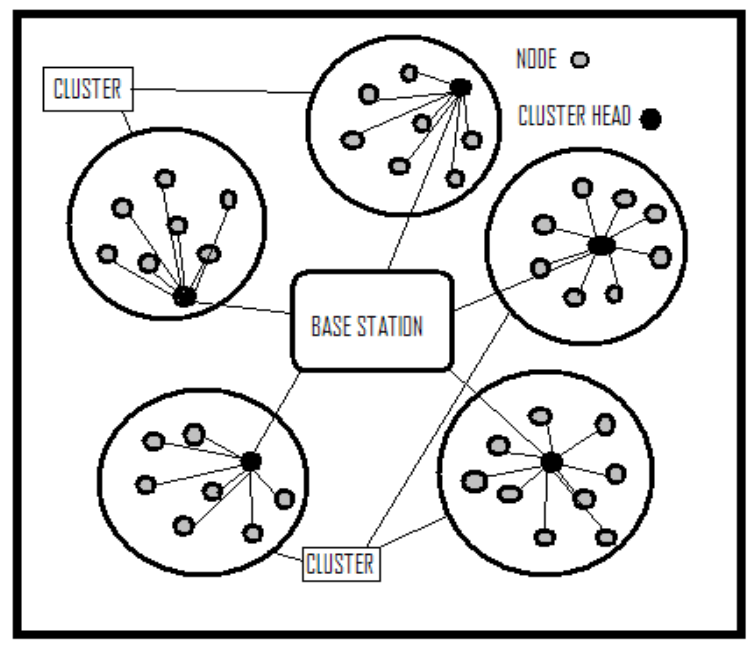

Fig.1: LEACH Protocol

However, in the entire network life cycle, these clusters and cluster-head nodes are fixed, and the local base station is assumed as a high-energy nodes situation. In most cases, the local base station is an energy-constrained node. The entire network may die soon because of excessive using about local base station node.

\subsection{Low-Energy Adaptive Clustering Hierarchy Protocol (LEACH)}

LEACH, which was presented by Heinzelman in 2000[10],[11] is a low-energy adaptive clustering hierarchy for WSN. The operation of LEACH can be divided into rounds. Each round begins with a set-up phase when the clusters are organized, followed by a steady state phase where several frames of data are transferred form the nodes to the cluster head and on to the base station. During the set-up phase, each sensor node tries to select itself as a cluster head according to probability model. Fig.2 demonstrates the LEACH protocol Phases.

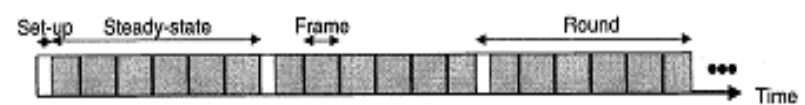

Fig.2:LEACH Protocol Phases
For selecting a cluster head, each sensor node generates a random number between 0 and 1 . If the number is less than the threshold $\mathrm{T}(\mathrm{n})$, the sensor node selects itself as a cluster head for current round, the threshold is presented as follows:

$\mathrm{T}(\mathrm{n})=[p /(1-p[\mathrm{r} \bmod (1 / p)])] \quad$ if $n € G$

\section{$0 \quad$ Otherwise}

where $p$ is the predetermined percentage of cluster heads (e.g., $p=$ $0.05), r$ is the current round, and $G$ is (e.g., $p=0.05$ ), $r$ is the current round, and $G$ is the set of nodes that have not been cluster heads in the last $1 / p$ rounds. Using this threshold, each node will be a cluster head at some round within $1 / p$ rounds. After $1 / p$ rounds, all nodes are once again eligible to become cluster heads. In LEACH, the optimal number of cluster heads is estimated to be about $5 \%$ of the total number of nodes. Each node that has elected itself a cluster head for the current round broadcasts an advertisement message to the rest of the nodes in the network. All the non cluster head nodes, after receiving this advertisement message, decide on the cluster to which they will belong for this round. This decision is based on the received signal strength of the advertisement messages. After cluster head receives all the messages from the nodes that would like to be included in the cluster and based on the number of nodes in the cluster, the cluster head creates a TDMA schedule and assigns each node a time slot when it can transmit. During the steady-state phase, the sensor nodes can begin sensing and transmitting data to cluster heads. The radio of each non-cluster head node can be turned off until the node's allocated transmission time. The cluster heads, after receiving all the data, aggregate it before sending it to the sink.

\subsection{Radio Model for Energy Calculation}

In this paper, we use the first order radio model. Here are some assumptions for our mechanism [12]. (1) All sensors are within the wireless communication range when they communicate with each other or with the BS. (2) All sensors have homogeneous sensing, computing and communication capabilities. (3) All sensors are randomly deployed in WSN. (4) BS is located in the center of the sensor networks and BS has infinity energy resource. (5) All sensors in the network have the same initial energy resource and dissipate their energy resource at the same rate. (6) Network lifetime is defined as the time span from the deployment to the instant when the first sensor dies (or when the entire sensors die). According to (5), all the sensors would exhaust their energy resource at the same time. (7) Both the energy dissipation of sensing data and the energy dissipation for clustering are neglected. Compared with the power consumption of CPU and Radio, the power consumption of sensor part is so small that can be neglected. Also, we suppose that all the clustering algorithms are run on the BS and no energy dissipation of clustering on sensor nodes. (8) The time span that BS collects the information from all the sensors once is defined as a round. In a round, each sensor has only one sensed data with the same packet size. (9) The 
sensors that receive the data combine one or more packets to produce a same-size resultant packet, and by this way, the number of data that need to send by radio is reduced. This is reasonable, because it is generally used to the scenario that there is much correlation among the data sensed by the different sensors. (10) The energy dissipation of fusing one bit data is a constant value. Therefore, the equations used to calculate transmission costs and receiving costs for a 1-bit message and a distance $\mathrm{d}$ are respectively shown in fig. 2(a).

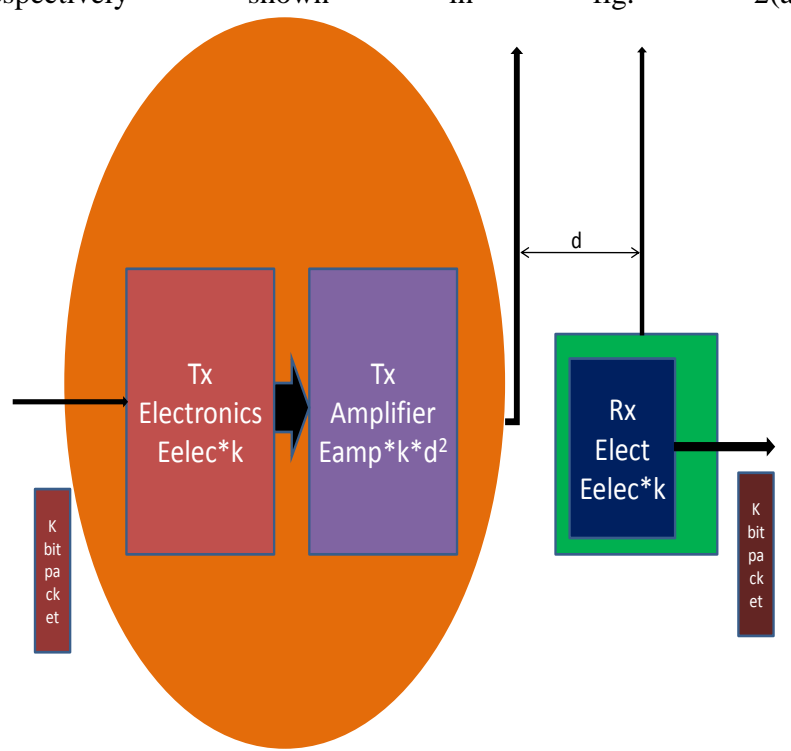

Fig.2a: First Order Radio Energy Model

Radio energy dissipation model adopted wireless channel models in the reference [11]. Thus, to transmit a 1-bit message a distance $\mathrm{d}$, the radio expends:

$$
k \text { Eelec }+k \varepsilon_{f s} d^{2} \quad d<d_{o}
$$

$E_{T x}(k, d)=$

$$
k \text { Eelec }+k \varepsilon_{m p} d^{4} \quad d \geq d_{o}
$$

The electronics energy, Eelec, depends on factors such as the digital coding, filtering and spreading of the signal, whereas the amplifier energy, $\varepsilon_{\mathrm{fs}}, \mathrm{d}^{2}, \varepsilon_{\mathrm{mp}}, \mathrm{d}^{4}$ depend on the distance to the receiver and the acceptable bit error rate and $d_{0}$ is a distance constant.

To receive this message, the radio expends:

$$
E_{R x}(d)=k \text { Eelec }
$$

\subsection{The network initialization for Homogeneous system}

The network includes some of the initial setting of energy parameters and the initialization of the sensor nodes. So it is necessary to generate a random distribution of these nodes in the
$\mathrm{L} * \mathrm{~L} \mathrm{~m} \mathrm{~m}^{2}$ of the region. Random 100 - node topology for a $100 *$ $100 \mathrm{~m}^{2 .}$, Sink is located at $(50,50)$. Fig. 3 demonstrates the wireless sensor network initialization for homogeneous system. Here all the available wireless sensor network nodes are having equal amount of initial energy $\mathrm{Eo}=0.5 \mathrm{~J}$.

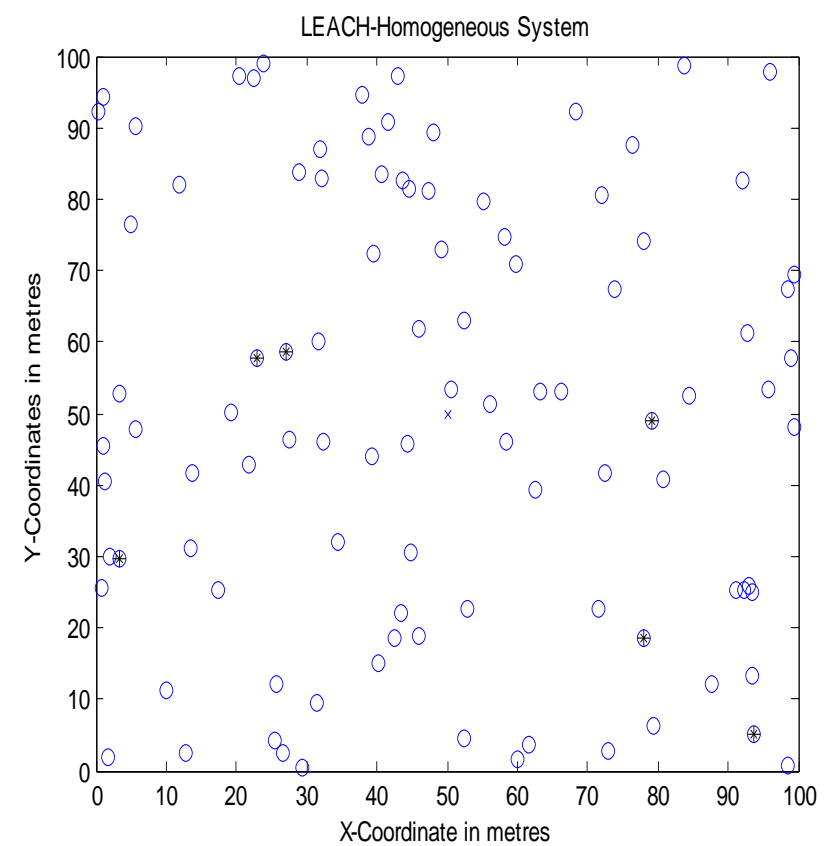

Fig.3: The wireless sensor network initialization

\section{PROPOSED PROTOCOL}

In this section we describe the proposed routing scheme which employs the Leach-Heterogeneous system in the network. The structure of the proposed Leach-Heterogeneous system for wireless sensor networks is shown in fig. 4. Here the same procedure as in the normal LEACH protocol is followed. By taking the number of sensor nodes shown in fig.4, the formation of the clusters is same in this heterogeneous system and also the cluster head selection by comparing the residual energy of the individual in every round[13]. In this Leach-Heterogeneous system 0.1 percentages of nodes are having more initial energy than the other nodes in the wireless sensor networks. For this case of Leach-Heterogeneous system 10 nodes are having 1 Joule of initial energy out of 100 nodes in the network. The remaining 90 nodes are having 0.5 joules of initial energy. When we increase the additional amount of energy to the nodes in the heterogeneous system of wireless sensor networks ultimately the additional energy are going to lost their energy in the end of the round. Depend the application, the number of advanced nodes can be increased and the total system lifetime can be increased significantly. 


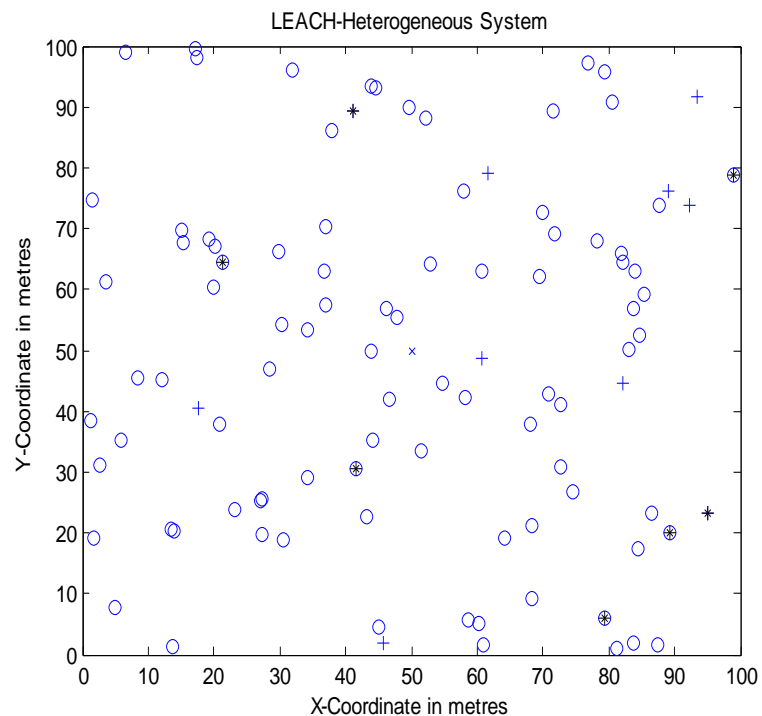

Fig.4: Leach-Heterogeneous system

\section{SIMULATION RESULTS}

In this section, we evaluate the performance of the proposed approach through the simulations. A simulator is designed and implemented in MATLAB in order to investigate the energy efficiency with lifetime extension of the mentioned protocol. We compare the proposed Leach-Heterogeneous system with LeachHomogeneous system. The simulation parameters used in the experiment is shown in Table I. The nodes are randomly distributed between $\mathrm{x}=0, \mathrm{y}=0$ and $\mathrm{x}=100, \mathrm{y}=100$ with the base station (BS) at location $x=50, y=50$. The fig. 5 and fig. 6 are shows total number of nodes that remain alive over simulation time of 1300 rounds for LEACH under homogeneous and heterogeneous system. It can be seen that nodes remains alive for a longer time (rounds) in proposed Leach-Heterogeneous system than LeachHomogeneous system. Note that further increasing of the number of nodes in the heterogeneous system and the area does improve the network lifetime considerably. Based on the simulation results, we found that an energy saving up to $40 \%$ is obtainable. Using the metrics, first node dies (FND) and Half of the nodes alive (HNA) in the proposed Leach-heterogeneous system is compared with Leach-Homogeneous system in terms of network lifetime.

Table 1: Used parameters details

\begin{tabular}{ll}
\hline \multicolumn{1}{c}{ Parameter name } & \multicolumn{1}{c}{ Values } \\
\hline Network area & $100 \mathrm{~m} * 100 \mathrm{~m}$ \\
Number of nodes & 100 \\
Initial Energy & $0.5 \mathrm{~J}$ \\
BS position & $50 \mathrm{~m} * 50 \mathrm{~m}$
\end{tabular}

Eelec

$50 \mathrm{~nJ} / \mathrm{bit}$

Etx $=$ Erx

$50 \mathrm{~nJ} / \mathrm{bit}$

$\varepsilon_{\mathrm{fs}}($ friss-amp)

$10 \mathrm{pJ} / \mathrm{bits} / \mathrm{m}^{2}$

$\varepsilon_{\mathrm{mp}}($ two-ray-amp)

$0.0013 \mathrm{pJ} / \mathrm{bit} / \mathrm{m}^{4}$

$\operatorname{do}=\operatorname{sqrt}\left(\varepsilon_{\mathrm{fs}} / \varepsilon_{\mathrm{mp}}\right)$

$\mathrm{E}_{\mathrm{DA}}$

$5 \mathrm{~nJ} / \mathrm{bit}$

Packet size

4000bits

Fig. 5 giving the simulation result of live nodes after 700 rounds for normal LEACH protocol. Here $50 \%$ of the nodes are lost their energy and nearly 45 nodes are in live position. When we have nearly 1500 rounds all the nodes in the network are lost their energy. But in the case of proposed protocol which is in the fig. 6 giving the simulation result of live nodes after 700 rounds are 94 nodes out of 100 nodes. This is indicated in figures 5 and 6 with blue round shape color as live nodes and red points are the energy lost nodes. The proportionate numbers of cluster head nodes are indicated as *.When we increase the topology size and the number of nodes the system lifetime of the network also proportionately increased ultimately the energy also efficiently consumed.

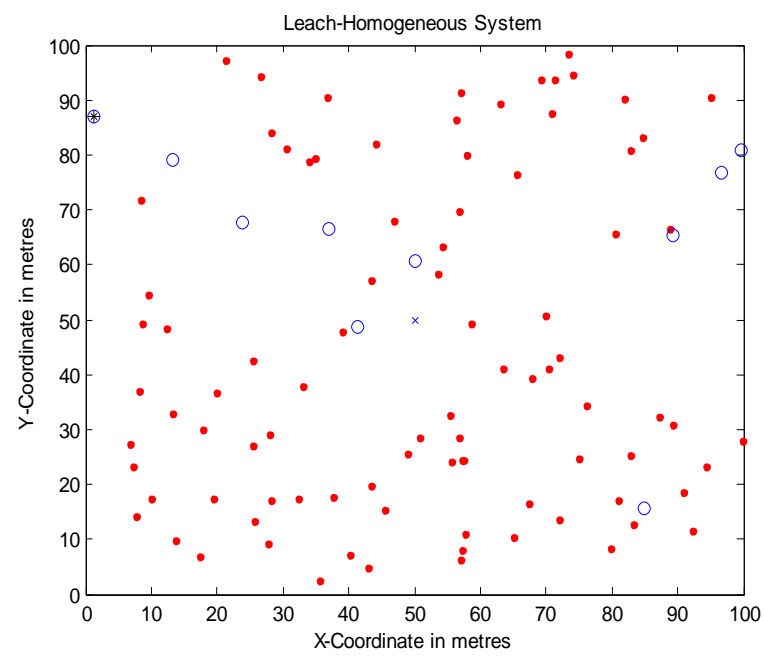

Fig.5: System lifetime using Leach-Homogeneous System after 1300 rounds. Red points are indicates dead nodes (Nearly 90 nodes). Cluster heads indicated as *. Sink node is at center as $\times$. 


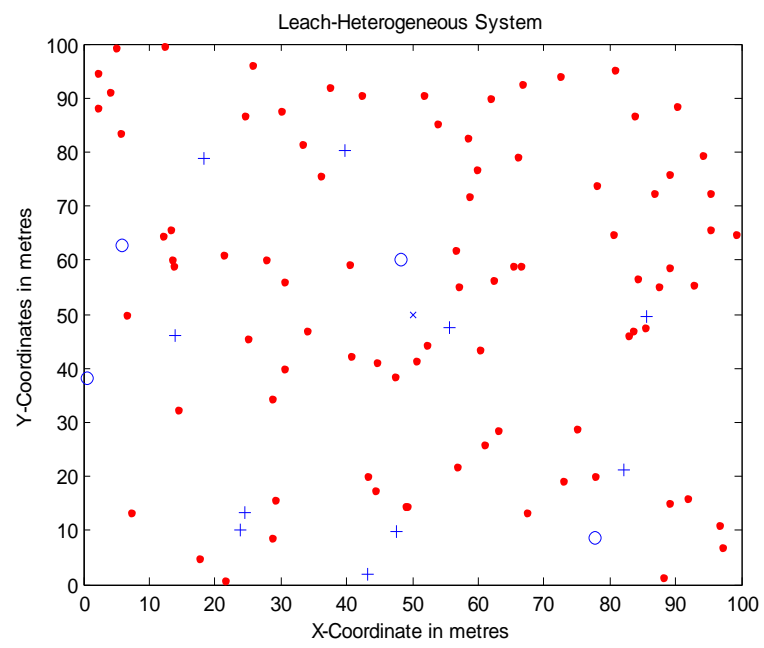

Fig.6: System lifetime using Leach- Heterogeneous System after 1300 rounds. Red points are indicates dead nodes (80 nodes). Cluster heads indicated as *. Sink node is at center as $\times$.Advance nodes with additional energy as + .

Fig. 7 describes the comparison between the LeachHomogeneous and Leach-Heterogeneous System. Here the total energy efficiency is increased nearly $40 \%$ than the Leach-

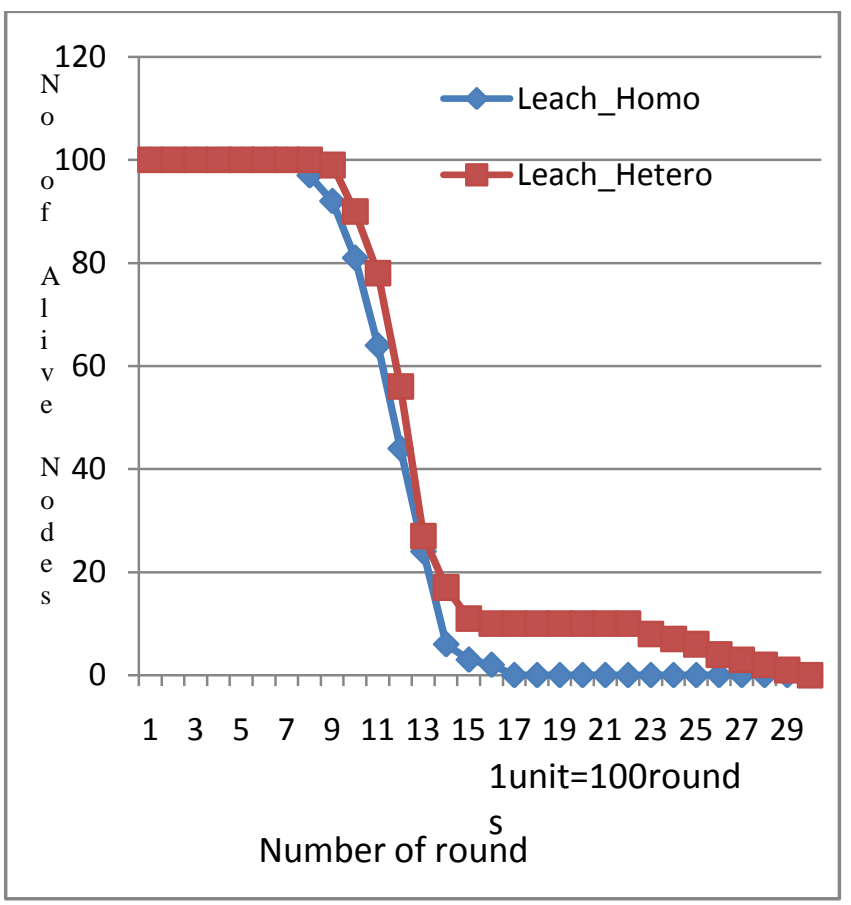

Fig.7: Total number of live sensor nodes Versus Number of rounds.
Homogeneous system. After 2700 rounds only the proposed Leach-Heterogeneous System sensor nodes are under dead position, but in the case of normal Leach-Heterogeneous System all the nodes are lost their energy nearly 1300 rounds itself. This is clearly giving a good result of the proposed LeachHeterogeneous System.

\section{CONCLUSION AND FUTURE WORK}

In this paper, we have presented the proposed Leachheterogeneous system in the individual clustering of the whole network, which is an energy efficient method for WSNs and compared it with the normal Leach- Homogeneous system. Results from our simulations using MATLAB are shows that the proposed Leach-Heterogeneous System provides better performance in energy efficiency and increasing level in lifetime of the wireless sensor networks.

For future work, a model with high density of heterogeneous wireless sensor nodes with its topology is proportionately increased according to the application to have good energy efficient and increasing lifetime network may be investigated. This may try to implement in ns 2 with stable and mobile mode of the system.

\section{REFERENCES}

[1] Mehdi Saeidmanesh,Mojtaba hajimohammadi, and Ali Movaghar, Energy and Distance Based Clustering: An Energy Efficient Clustering Method for WSNs, World Acadamy of science, Engineering and Technology 552009

[2] Saravanakumar.R, Kavitha.M, Performance Evaluation of energy aware data centric routing algorithm in wireless sensor networks, National level conference on Intelligent systems and their applications held at Sudharsan Engineering College pudukkottai,Tamilnadu, India during $24^{\text {th }}$ and $25^{\text {th }}$, August-2007.

[3] Saravanakumar.R, Susila.S.G, iPower- An energy conservation system for intelligence building by WSNs.International Conference on Data Warehousing, Data Mining and Networking held at Jayaram College of Engineering and Technology,Tiruchirappalli,Tamilnadu, India during $4^{\text {th }}$ and $5^{\text {th }}$ October 2007.

[4] Saravanakumar.R,Malaisamy.K, Energy-Efficient MAC for WSNs.National conference on Application of Emerging Technologies held at Adhiyaman College of Engineering ,Hosur,Tamilnadu, India during $24^{\text {th }}$ and $25^{\text {th }} 2008$.

[5] Eugenio M., "A Survey on Wireless Sensor Networks", www-lia.deis.unibo.it/Courses/RetiLS/.

[6] Muruganathan S. D., F Ma. D. C., Bhasin R. I., and Fapojuwo A. O., "A Centralized Energy-efficient Routing Protocol for Wireless Sensor Networks", IEEE Communications Magazine. 2005, pp. S8 - S13. 
[7] HaiGang GONG, et a1, Distributed Energy Efficient Data Gathering in WirelessSensor Networks, ACTA ELECTRONICA SINICA, 2005.8.

[8] Bo Shen, et al, Cluster-Based Routing Protocols for Wireless Sensor Networks, Journal of Software, 2006.7.

[9] Gang Hu, et al. Research and Improvement of LEACH for Wireless Sensor Networks. Chinese Journal of Sensors and Actuators 2007.6.

[10] W.R.Heinzelman, Energy-efficient Communication protocol for Wireless microsensor networks, In: Proc. of $33^{\text {rd }}$ Annual Hawaii Inter Cord on System Sciences, Hawaii, USA: IEEE Computer Society, 2000.

[11] Heinzelman W. B., Chandrakasan A. P., and Balakrishnan H, "An Application-specific Protocol Architecture for Wireless Micro sensor Networks", IEEE Trans. on Wireless Communications, 2002, pp.660-670.

[12] Yan Li, YanZhong Li, Energy-Efficient clustering Routing algorithm based on LEACH, Journal of Computer Applications 2007.

[13] Gang Hu, et al. Research and Improvement of LEACH for Wireless Sensor Networks. Chinese Journal of Sensors and Actuators 2007.6.

\section{AUTHORS PROFILE}

R.Saravanakumar is currently doing his research in Faculty of Information and Communication Engineering, Anna University of
Technology Tiruchirappalli. Tiruchirappalli Tamilnadu. India. and also working as Professor, Department of Electronics and communication Engineering in Pavendar Bharathidasan College of Engineering, Tiruchirappalli,Tamilnadu, India. He received his B.E (ECE) and M.E (Communication Systems) from Bharathidasan University, Tiruchirappalli,Tamilnadu, India in 1995 and 2001.

S.G.Susila is currently working as faculty of Electronics and Communication Engineering, Anna University of Technology, Tiruchirappalli, Tiruchirappalli Tamilnadu, India. She received her B.E (ECE) and M.E (Communication Systems) from Bharathidasan University,Tiruchirappalli and Anna university Chennai, Tamilnadu, India in 1996 and2006 respectively. She presented five papers in national/ international conferences. She is doing her research in the field of wireless sensor networks.

Dr.J.Raja is working as Professor and Head of Department of Electronics and Communication Engineering and Registrar in Anna University of Technology Tiruchirappalli, Tiruchirappalli Tamilnadu, India.He received his B.E. (ECE) from Bharathiyar University in 1988. M.E. (C\&I) in 1992 and Ph.D. from Anna University, Chennai in 2003 .He pursued his research in the area of ATM networks. Guided two Ph.D. theses and currently he is guiding 7 research scholars in the areas of Error Control Coding, WSN Routing Techniques. He has Published 11 International/ National journal papers and 38 International and National conference papers 